\title{
Protección de acero para instrumentos quirúrgicos con recubrimientos duros en base carbono tipo DLC
}

\author{
Eugenia Laura Dalibon Bähler ${ }^{1}$,Jorge Nahuel Pecina ${ }^{1}$,

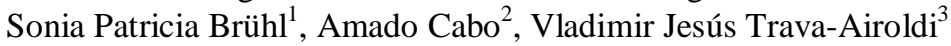

\author{
${ }^{1}$ Grupo de Ingeniería de Superficies, Facultad Regional Concepción del Uruguay, Universidad Tecnológica Nacional, \\ Ingeniero Pereira 676, E3264BTD, Entre Ríos, Argentina, Argentina. \\ e-mail: eugedalibon@yahoo.com.ar,nahuelpecina@gmail.com, sonia@frcu.utn.edu.ar \\ ${ }^{2}$ Ionar S.A. Arias 3422, 1430, Buenos Aires, Buenos Aires, Argentina \\ e-mail: cabo@ionar.com.ar \\ ${ }^{3}$ Instituto Nacional de Pesquisas Espaciáis - INPE,Av. dos Astronautas 1758, 12.227-010 São José dos Campos, SP, Bra- \\ sil. \\ e-mail: vladimir.airoldi@inpe.br
}

\section{RESUMEN}

Los aceros inoxidables martensíticos como el AISI 420 tienen múltiples aplicaciones, entre ellas instrumentos quirúrgicos, donde se requiere buena resistencia al desgaste y a la corrosión. Los tratamientos de modificación superficial, como la nitruración iónica, permiten mejorar las propiedades mecánicas de estos aceros pero afectan su resistencia a la corrosión. Por esta razón, un tratamiento posterior de deposición de recubrimiento tipo DLC ("Diamond Like Carbon", carbono símil diamante) puede ser conveniente. En este trabajo se estudió el comportamiento al desgaste y a la corrosión de muestras dúplex (nitruradas y recubiertas por PACVD) y se compararon con muestras nitruradas, sólo recubiertas, nitruradas y sólo templadas y revenidas. Se analizaron los recubrimientos con espectroscopia Raman y EDS. Se realizaron ensayos de desgaste tipo "pin on disk" y se evaluó la adhesión mediante ensayos de Scratch Test e indentación Rockwell C. Se midió nanodureza con indentador Berkovich. Se llevaron a cabo los ensayos propuestos para instrumentos quirúrgicos en la Norma F 1089-02 (prueba de agua hirviendo y de hisopado con sulfato de cobre) y ensayos de niebla salina.

Estos recubrimientos tienen una relación $\mathrm{I}_{\mathrm{D}} / \mathrm{I}_{\mathrm{G}}$ de 0,8 y bajo contenido de hidrógeno aproximadamente del $20 \%$. El espesor de los recubrimientos fue de $2 \mu \mathrm{m}$ y la dureza alcanzó $24 \mathrm{GPa}$. La capa nitrurada tuvo un espesor de $10 \mu \mathrm{m}$. Las muestras dúplex y recubierta presentaron bajo coeficiente de fricción y el volumen desgastado fue indetectable. Además, tuvieron buen comportamiento a la corrosión en el ensayo de niebla salina y en los ensayos propuestos en la Norma ASTM F 1089-02. No obstante, la muestra sólo nitrurada presentó una región corroída en el ensayo de sulfato de cobre y en el de agua hirviendo. La muestra dúplex presentó mejor adhesión que la muestra sólo recubierta en los ensayos de Scratch Test y de indentación Rockwell C.

Palabrasclave: DLC coatings, AISI 420, desgaste, corrosión, instrumentos quirúrgicos

\section{ABSTRACT}

Martensitic stainless steels can be used in different applications such as surgical instruments where a good wear and corrosion resistance are required. Surface modification treatments such as nitriding improve the mechanical properties of these steels, however; they affect the corrosion resistance. For this reason, the DLC (Diamond Like Carbon) coatings deposition can be convenient.

The wear and corrosion behaviour was studied in duplex samples (nitrided and PACVD coated) and they were compared with nitrided and only coated samples and with those without treatment, only heat treated (quench and tempering). The coatings were analyzed by Raman spectroscopy and EDS. Pin on disk tests were performed and the adhesion was evaluated using Scratch Test and Rockwell C Indentation. The nanohardness was measured with a Berkovich indenter. The corrosion tests for surgical instruments (according to the Standard ASTM F 1089 -02) and Spray Salt Fog tests were carried out.

In the DLC coatings, the $\mathrm{I}_{\mathrm{D}} / \mathrm{I}_{\mathrm{G}}$ ratio was 0.8 and the hydrogen content was calculated and resulted to be about 
$20 \%$. The coating thickness was $2 \mu \mathrm{m}$ and the hardness reached $24 \mathrm{GPa}$. The nitrided layer thickness was 10 $\mu \mathrm{m}$. The duplex and coated samples presented low friction coefficient and the wear volume loss was undetectable. The coatings had good corrosion behaviour in the corrosion tests. Nevertheless, the nitrided sample presented a corroded region in the copper sulfate and boil tests. The duplex samples had better adhesion than the coated samples in scratch test and indentation Rockwell C.

Keywords: DLC coatings, AISI 420, wear, corrosion, surgical instruments.

\section{INTRODUCCIÓN}

Los aceros inoxidables martensíticos tienen múltiples aplicaciones, entre ellas, como materiales de instrumentos quirúrgicos. Estos utensilios son costosos, sofisticados y deben someterse al proceso de descontaminación, limpieza y esterilización con frecuencia [1]. Deben tener propiedades específicas para hacerlos resistentes a la corrosión cuando se exponen a sangre y líquidos corporales, soluciones de limpieza y a la atmósfera, dado que es importante asegurar el buen uso del instrumental y su conservación para incrementar su vida útil [1]. Se requiere resistencia a la fatiga, propiedades de barrera para prevenir la corrosión, resistencia al daño en el ambiente clínico para evitar el desgaste o la rotura durante los procedimientos normales de limpieza y operación. Además, baja fricción superficial para disminuir la adhesión de biopelículas y aumentar la efectividad del régimen de limpieza post-operativo[2-4]. Para mejorar las propiedades superficiales de los materiales, se pueden realizar diferentes tratamientos de modificación superficial como la nitruración. Este tipo de proceso permite mejorar las propiedades tribológicas (incluyendo resistencia a la fatiga), pero dependiendo de las condiciones de ensayo puede afectar la resistencia a la corrosión del acero debido a la precipitación de nitruros de cromo que reducen el cromo en solución para formar el óxido pasivante [5-7]. Para mejorar aún más las propiedades superficiales, se pueden depositar recubrimientos DLC, es decir carbono símil diamante ("Diamond Like Carbon" en inglés),que presentan bajo coeficiente de fricción, buena resistencia al desgaste y son químicamente inertes [8-10].

El tratamiento combinado de nitruración + recubrimientos (proceso dúplex) podría ser una buena opción para aumentar la vida útil de los instrumentos quirúrgicos de acero inoxidable. En este se combinan las buenas propiedades tribológicas y anticorrosivas del recubrimiento, con el perfil de dureza en profundidad y la resistencia a la fatiga de la capa nitrurada, mejorando la capacidad de soporte de carga y la resistencia a la deformación del sistema. Consecuentemente, mejora el comportamiento mecánico del mismo [11-13].

Si bien hay registros en la literatura de estudios realizados sobre el uso de recubrimientos tipo DLC (debido a sus propiedades anti-adhesivas y bajo coeficiente de fricción) sobre aceros inoxidables para instrumentos quirúrgicos[2-4], no hay experiencias usando la nitruración como pre tratamiento, y cada sustrato y recubrimiento requiere un estudio específico.

En este trabajo se estudia el comportamiento frente al desgaste y a la corrosión del acero inoxidable martensítico AISI 420 nitrurado y sin nitrurar, teniendo en cuenta los requerimientos de los instrumentos quirúrgicos según la Norma ASTM F 1089-02 [14]. Además se evaluó la adhesión teniendo en cuenta la presencia de la capa nitrurada previa a la deposición del recubrimiento.

\section{MATERIALES Y MÉTODOS}

El material utilizado en este estudio fue el acero inoxidable martensítico AISI 420, cuya composición química (porcentaje en peso) es 0,38\% C, $13 \% \mathrm{Cr}, 0,44 \% \mathrm{Mn}, 0,42 \% \mathrm{Si}, 0,07 \% \mathrm{Mo}, 0,02 \% \mathrm{P}$ y Fe como balance. Las muestras utilizadas se cortaron de una chapa de $2 \mathrm{~mm}$ de espesor en discos de $25 \mathrm{~mm}$ de diámetro y fueron templadas y revenidas. El tratamiento térmico se realizó dela siguiente manera: las probetas fueron sometidas a un precalentamiento a $800^{\circ} \mathrm{C}$ durante 15 minutos, luego se llevaron a $1030^{\circ} \mathrm{C}$ durante 30 minutos, fueron templadas en aire agitado, y se realizaron dos revenidos a $260^{\circ} \mathrm{C}$ durante 2 horas.

Los tratamientos de nitruración se realizaron en el equipo industrial de Ionar S.A. con una descarga DC pulsada, en una mezcla gaseosa de $20 \% \mathrm{~N}_{2}-80 \% \mathrm{H}_{2}$, a $390^{\circ} \mathrm{C}$ durante 10 horas. Los recubrimientos DLC fueron depositados por PACVD (Plasma Assisted Chemical Vapor Deposition) utilizando acetileno como gas precursor, a una temperatura de $150^{\circ} \mathrm{C}$ durante 2 horas. Previo a la deposición del recubrimiento se depositó una capa de silicio amorfo para mejorar la adhesión[14]. Los recubrimientos fueron depositados sobre muestras de acero inoxidable AISI 420 nitrurado (muestra dúplex) y sin nitrurar (muestra recubierta).

El recubrimiento DLC se caracterizó por EDS y Espectroscopía Raman. Se analizó su microestructura y la de la capa nitrurada por microscopia óptica, SEM-FIB y difracción de rayos X. Se realizaron ensayos de pin on disk (bolilla contra disco plano) con los siguientes parámetros de ensayo: $500 \mathrm{~m}$ de recorrido, carga de $5 \mathrm{~N}$, radio de huella de $7 \mathrm{~mm}$, contraparte de alúmina y velocidad tangencial de $10 \mathrm{~cm} / \mathrm{s}$. 
Para estudiar el comportamiento a la corrosión se realizaron ensayos de niebla salina según la Norma ASTM B117 [16]. Se realizaron las pruebas de agua hirviendo y de sulfato de cobre de acuerdo a la Norma F 1089-02. La prueba de agua hirviendo consiste en sumergir las muestras en un recipiente con agua destilada, calentar el agua hasta que hierva y dejar las muestras allí durante 30 minutos, posteriormente quitar la fuente de calor y dejar las muestras sumergidas durante 3 horas. La prueba de sulfato de cobre consiste en colocar sobre la muestra una gota de la solución y esperar 6 minutos para observar si deposita o no. Además se realizaron ensayos potenciostáticos utilizando distintos escalones en una solución de $3.5 \% \mathrm{NaCl}$. Los escalones de potencial fueron de $200 \mathrm{mV}$ y una duración de $1800 \mathrm{~s}$ cada uno. Las superficies fueron observadas con microscopio óptico después de cada ensayo. Para evaluar la adhesión se realizaron ensayos de indentación Rockwell C, con $300 \mathrm{~N}$ de carga estática. Finalmente, se realizaron ensayos de Scratch Test con carga constante de $20 \mathrm{~N}$ y $35 \mathrm{~N}$.

\section{RESULTADOS Y DISCUSIÓN}

\subsection{Caracterización del recubrimiento y de la capa ntirurada}

En la Figura 1, se representa el espectro Raman correspondiente al recubrimiento DLC. En dicho espectro, se detectaron las bandas $\mathrm{D}$ y $\mathrm{G}$ características de este tipo de recubrimientos. La relación de $\mathrm{I}_{\mathrm{D}} / \mathrm{I}_{\mathrm{G}}$ fue de 0,8 . Teniendo en cuenta este valor y la posición de la banda $\mathrm{G}$ podría indicarse que el porcentaje de uniones $\mathrm{sp}^{3}$ C-C alcanzó aproximadamente el 10\% según el modelo de tres etapas propuesto por Ferrari et al.[15]. A partir de la pendiente de la línea de ajuste a la base del espectro original, se pudo determinar que el porcentaje de hidrógeno es del $12 \pm 2 \%$ aproximadamente[16].

Los eespectros de EDS (Figura 2), indican presencia de carbono y silicio en las muestras, provenientes del recubrimiento y de la intercapa respectivamente, como era esperado.

Con los datos anteriormente mencionados se puede identificar que el recubrimiento es de tipo a:C-H, es decir carbono amorfo hidrogenado, pero con un bajo contenido de hidrógeno.

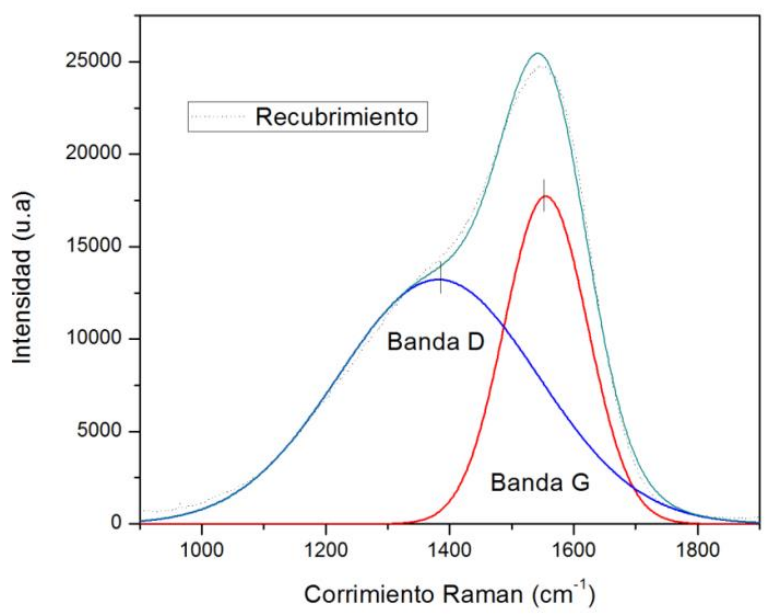

Figura 1: Espectro Raman del recubrimiento DLC

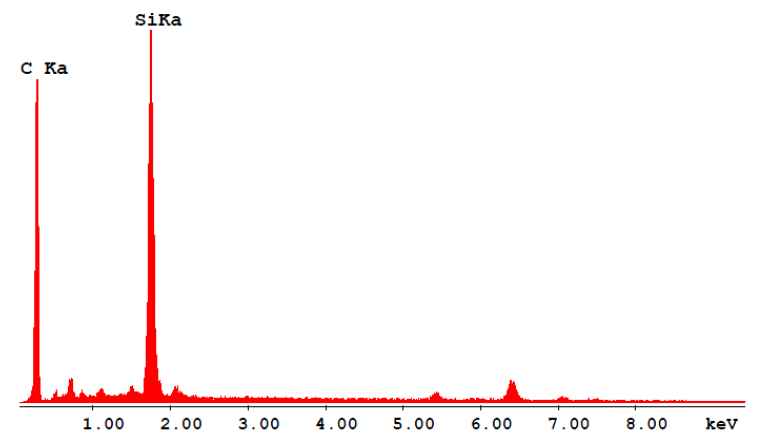

Figura 2: Espectro EDS del recubrimiento DLC. 
El recubrimiento resultó de 1,4 $\mu \mathrm{m}$ de espesor incluyendo la intercapa de silicio que se depositó previo al recubrimiento, presentó una interfase bien definida y regular con el sustrato, tanto en la muestra sólo recubierta como en la muestra dúplex. En la Figura 2 se observa una sección transversal obtenida con el haz de iones en un SEM-FIB. Previamente al corte, la zona se recubrió con una capa de platino (Figura 3).

La capa nitrurada resultó aproximadamente $(10 \pm 1) \mu \mathrm{m}$ de espesor, medido en la micrografía óptica después de atacarla probeta con el reactivo de Vilella (no mostrada). No se observaron zonas oscuras, que usualmente indican una precipitación masiva de nitruros de cromo [17]. La capa nitrurada presentó una interfase no bien definida con el material base de estructura martensítica como es característico en este tipo de aceros $[6,17]$. De acuerdo a los análisis realizados por DRX, en la muestra recubierta se detectaron los picos de martensita del acero templado y revenido debido a que el recubrimiento es amorfo y transparente a los rayos X. En la muestra dúplex, se detectaron los picos de martensita expandida por nitrógeno, más ensanchados y dislocados hacia ángulos $2 \theta$ menores, indicando que la red está expandida y tensionada con respecto al material sin tratar [5-7] tal como se muestra en la figura 4. Además, si bien nitruros de hierro fueron detectados, no se observó la presencia de nitruros de cromo [7] .

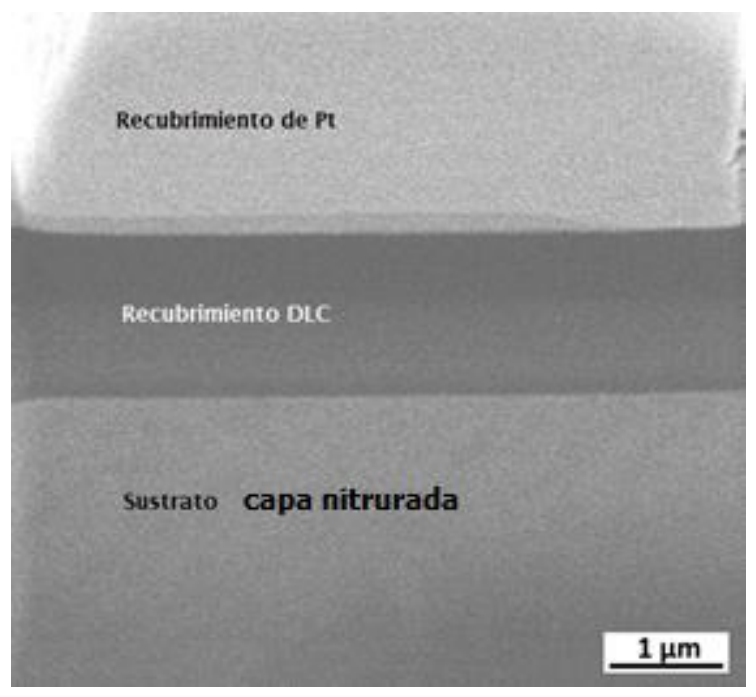

Figura 3: Imagen SEM-FIB del recubrimiento en la muestra dúplex, es decir, muestra nitrurada y recubierta con DLC.

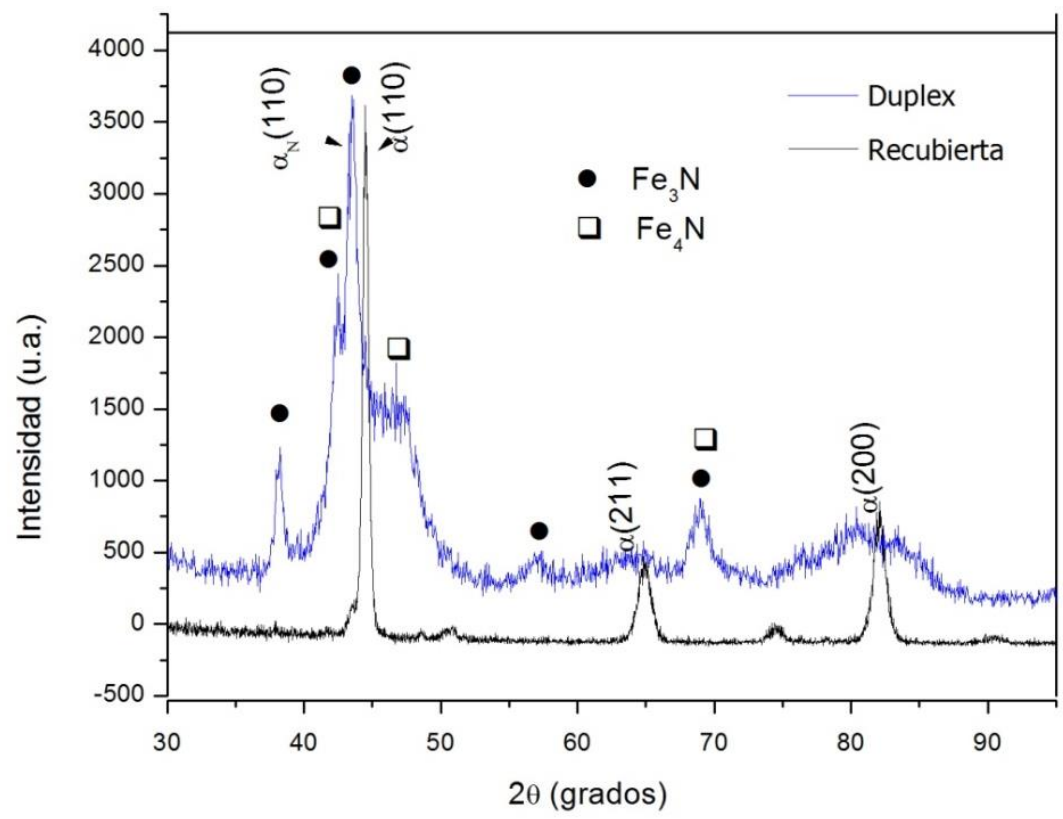

Figura 4: Difractogramas de Rayos X correspondientes a las muestras dúplex y recubierta. 


\subsection{Propiedades mecánicas}

La dureza del recubrimiento medida con nanoindentador Berkovich fue de $(24,5 \pm 1,5) \mathrm{GPa}$. Este valor de dureza corresponde al recubrimiento, debido a que la profundidad de penetración de la impronta no superó el

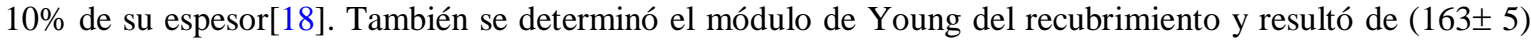
GPa. El valor de dureza del recubrimiento confirma que éste es de tipo duro o "hard DLC", como se lo nombra en la literatura internacional[8]. La dureza de la capa nitrurada resultó de (1066 27) $\mathrm{HV}_{0,05}$ y la del acero sin tratamiento, $(550 \pm 20) \mathrm{HV}_{0,05}$.

En los ensayos de pin on disk, las muestras recubiertas presentaron bajo coeficiente de fricción, aproximadamente 0,1 (Figura 5) y las muestras nitruradas y patrón presentaron un valor estable del coeficiente de fricción de 0,8 . Se puede apreciar que la presencia del recubrimiento reduce el coeficiente de fricción notablemente con respecto a la muestra nitrurada y patrón. Esto se debe a que se forma una capa de características grafíticas que tiene un efecto lubricante, como se ha reportado previamente en la literatura[8,9,19]. En cuanto al volumen desgastado, la profundidad máxima de la huella de desgaste en las muestras recubierta y dúplex resultó un orden de magnitud menor que en la muestra nitrurada. La profundidad máxima en la muestra recubierta fue del orden de $0,1 \mu \mathrm{m}$ y mientras que en la nitrurada de 1,3 $\mu \mathrm{m}$ como puede observarse en la Figura 6.

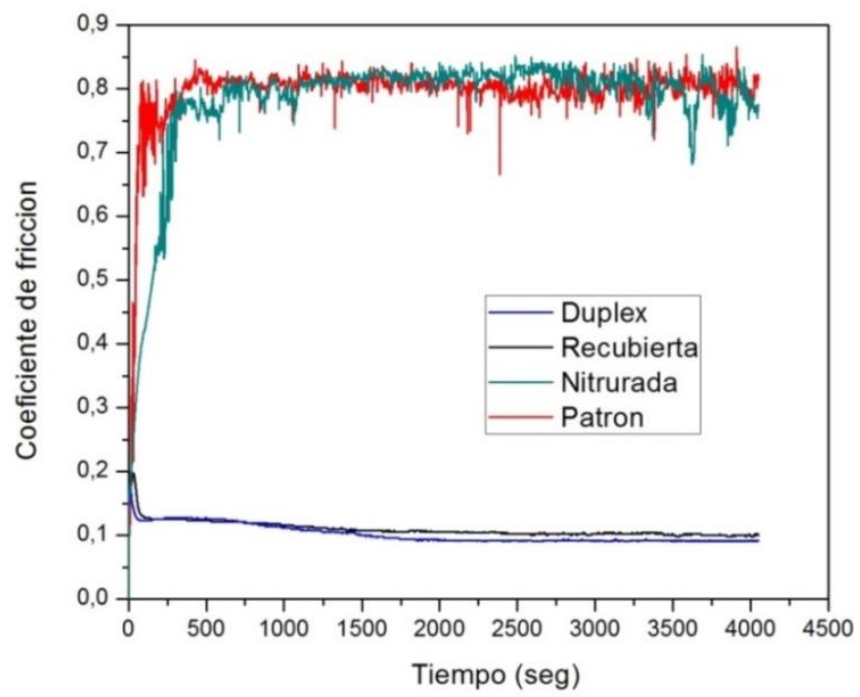

Figura 5: Coeficiente de fricción en función del tiempo del ensayo de Pin on Disk correspondiente a las diferentes muestras estudiadas.

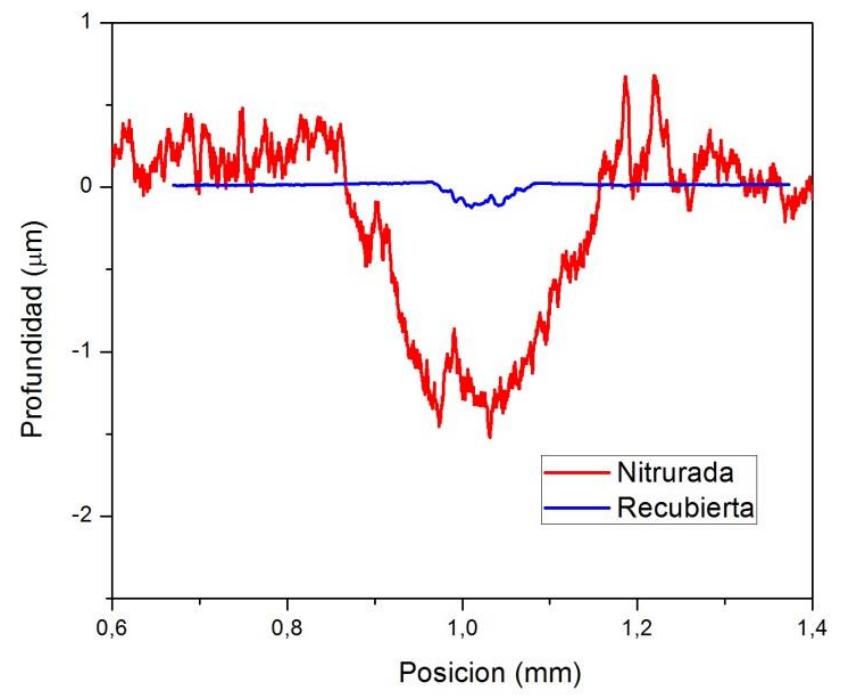

Figura 6: Perfiles de la huella de desgaste de las muestras recubierta y nitrurada. La posición de $1 \mathrm{~mm}$ corresponde al centro del punto de contacto. 


\subsection{Comportamiento a la corrosión}

En los ensayos de niebla salina, las muestras dúplex y recubierta no presentaron signos de corrosión general ni localizada. Las muestras nitruradas, y las templadas y revenidas presentaron una región de corrosión general próxima al borde. En la muestra nitrurada, es posible que la capa nitrurada sea diferente en espesor, composición y dureza en los bordes respecto del centro debido a lo que se conoce como "efecto de borde" en la nitruración por plasma, donde es la pieza de trabajo la que actúa como cátodo. Esto ya ha sido reportado en la literatura [20]. Además, la región del borde es más sensible a la corrosión debido a que las muestras fueron cortadas utilizando una mecha copa, la cual produce una gran cantidad de deformación plástica en los mismos.
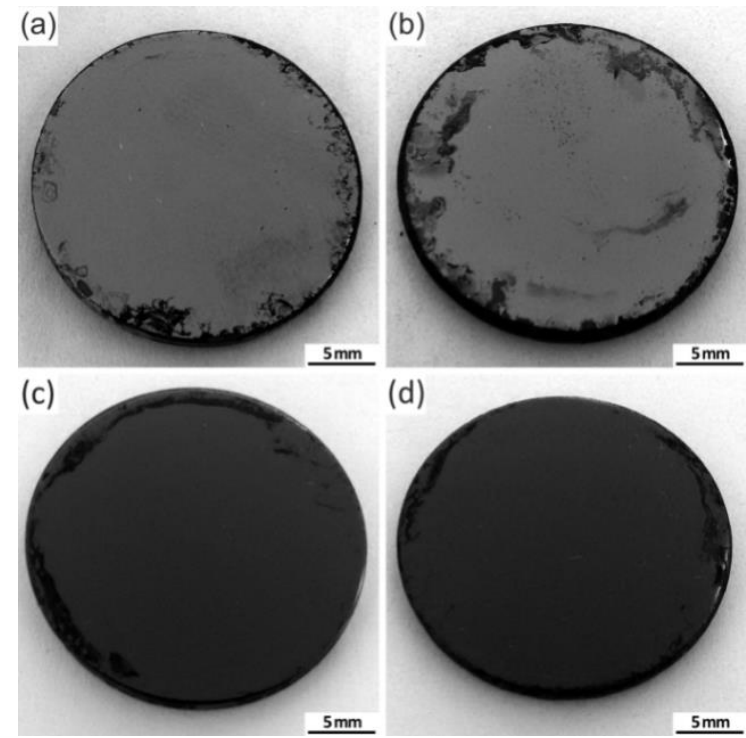

Figura 7: Imágenes de la superficie de las muestras posteriormente al ensayo de niebla salina según la Norma ASTM B1117: a) muestra patrón, b) muestra nitrurada, c) recubierta, d) dúplex.

En el ensayo de agua hirviendo de acuerdo a la Norma ASTM F1089-02, las muestras recubiertas no presentaron signos ni de corrosión general ni localizada como puede observarse en la Figura 8.
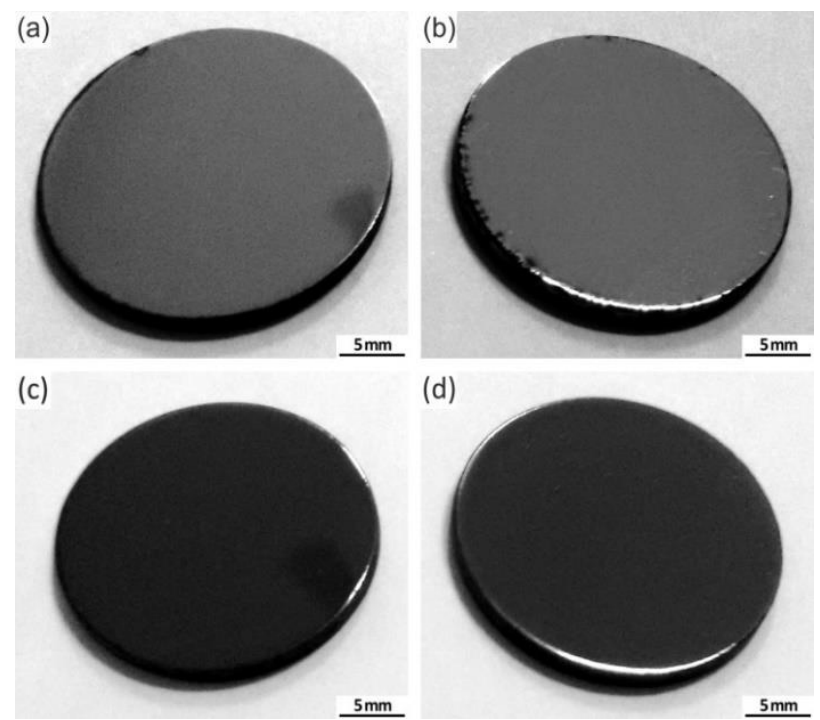

Figura 8: Imágenes de la superficie de las muestras posteriormente al ensayo de agua hirviendo según la Norma F108902: a) muestra patrón, b) muestra nitrurada, c) recubierta, d) dúplex. 
Sin embargo, también se observa en la Figura 8 que, tanto la muestra patrón como la nitrurada presentaron una pequeña región corroída en el borde como se muestra a continuación. Estos signos pueden ser considerados aceptables de acuerdo a lo establecido en la Norma.

En el ensayo de sulfato de cobre realizado de acuerdo a la Norma ASTM F1089-02, las muestras recubiertas no presentaron ningún ataque corrosivo o deposición de cobre después de realizar la prueba de hisopado con la solución de sulfato de cobre. Por otro lado, en la muestra nitrurada sí se produjo la deposición de cobre, indicando que hay hierro libre, es decir que la capa pasiva no se formó correctamente o que presenta alguna discontinuidad (Figura9). Este comportamiento podría indicar que hubo una reducción de cromo en solución, impidiendo que se formara la capa pasiva. A pesar de que el tratamiento de nitruración se realizó a baja temperatura, es posible que hayan precipitado nitruros de cromo (no detectados por difracción de rayos $\mathrm{X})$ que reducen la cantidad de cromo en solución para formar el óxido pasivante [6,21].

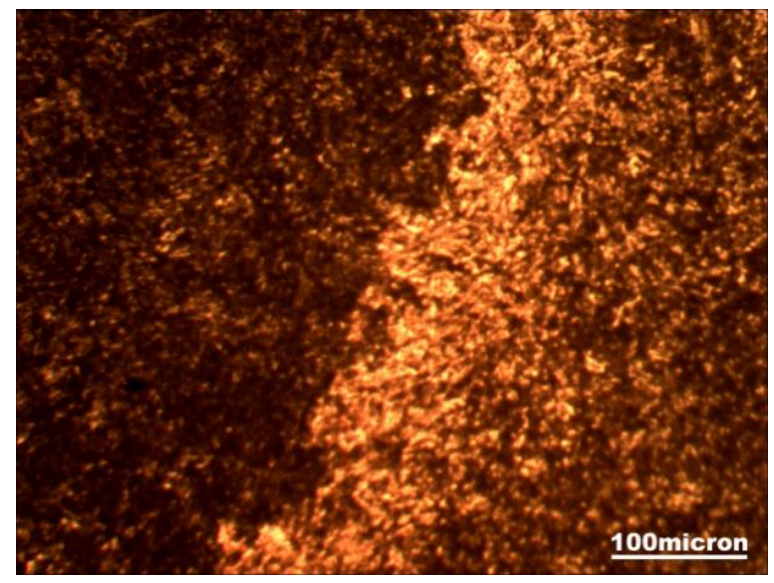

Figura 9: Micrografía óptica de la superficie de la muestra nitrurada después de la prueba de sulfato de cobre.

Se muestran en la Figura 10 algunas curvas representativas de la densidad de corriente en función del tiempo correspondientes a la muestra patrón, dúplex y recubierta, obtenidas de los ensayos potenciostáticos realizados.

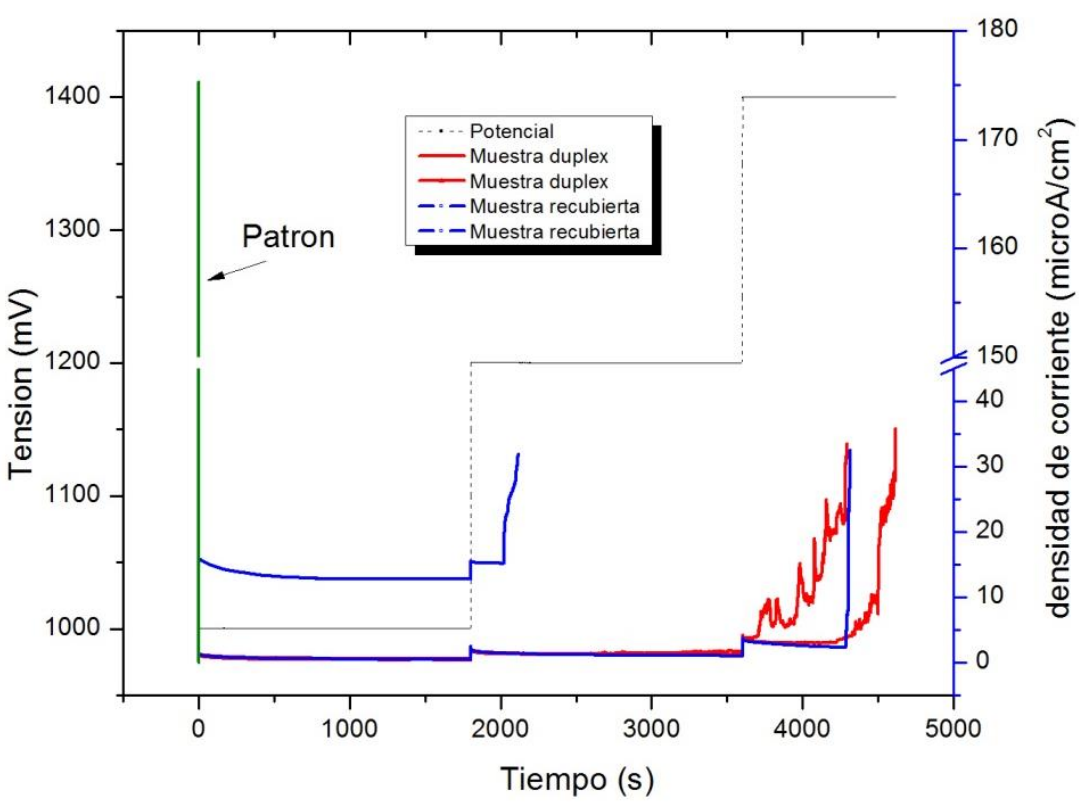

Figura 10: Curvas de los ensayos potenciostáticos para las muestras dúplex, recubierta y patrón. 
Puede observarse que para a muestra dúplex, la densidad corriente se mantiene en un valor muy bajo y aproximadamente constante hasta el escalón correspondiente a $1400 \mathrm{mV}$ y luego presenta un crecimiento abrupto. En las muestras recubiertas, la densidad de corriente crece abruptamente para un potencial algo menor que varía entre $1200 \mathrm{mV}$ y $1400 \mathrm{mV}$, pero presentan un comportamiento similar al de la muestra dúplex.

En la muestra patrón, en cambio, apenas se aplica el potencial correspondiente al primer escalón de $1000 \mathrm{mV}$, la densidad de corriente se eleva abruptamente, alcanzando un valor tres veces mayor que el correspondiente a las muestras recubiertas.

El recubrimiento mejora notablemente el comportamiento a la corrosión en este tipo de ensayos, comportándose como un aislante, barrera para la corrosión para un rango de tensión determinado. Es probable que el incremento de la densidad de corriente esté vinculado con la presencia de defectos en la zona ensayada, como se observa en la Figura 11.
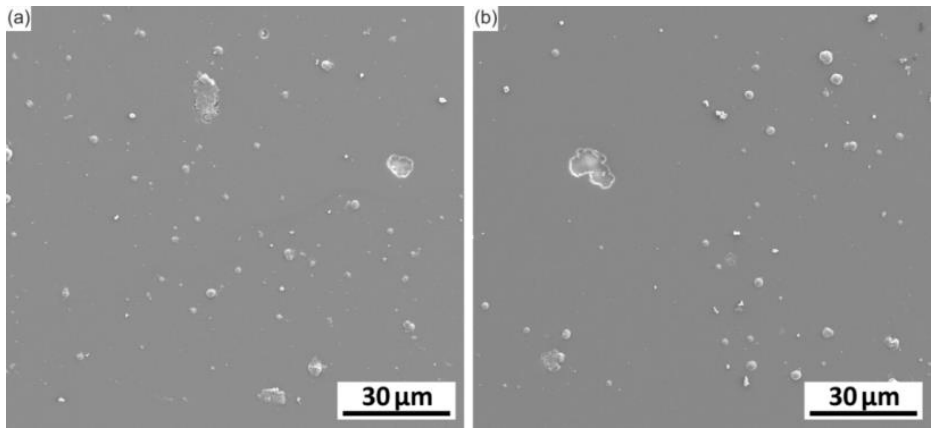

Figura 11: Imágenes SEM de la superficie del recubrimiento previo a los ensayos de corrosión a) y b) corresponden a dos regiones diferentes.

En la Figura 11, se puede observar que los recubrimientos presentan diferentes tipos de imperfecciones: protuberancias y discontinuidades o huecos. Probablemente, son éstos últimos los que tienen mayor influencia en el comportamiento a la corrosión, dado que permiten que la solución corrosiva alcance al sustrato.

\subsection{Adhesión}

Con respecto a la adhesión, como puede observarse en las micrografías (Figura 12), en la muestra recubierta parte del recubrimiento se desprendió después de realizarse el ensayo de Scratch Test con una carga de $20 \mathrm{~N}$, en cambio, la muestra dúplex no presentó desprendimiento para la misma carga. Al aplicar una carga de $35 \mathrm{~N}$, en ambas muestras el recubrimiento se desprendió, no obstante resultó mayor el daño en la muestra sólo recubierta.

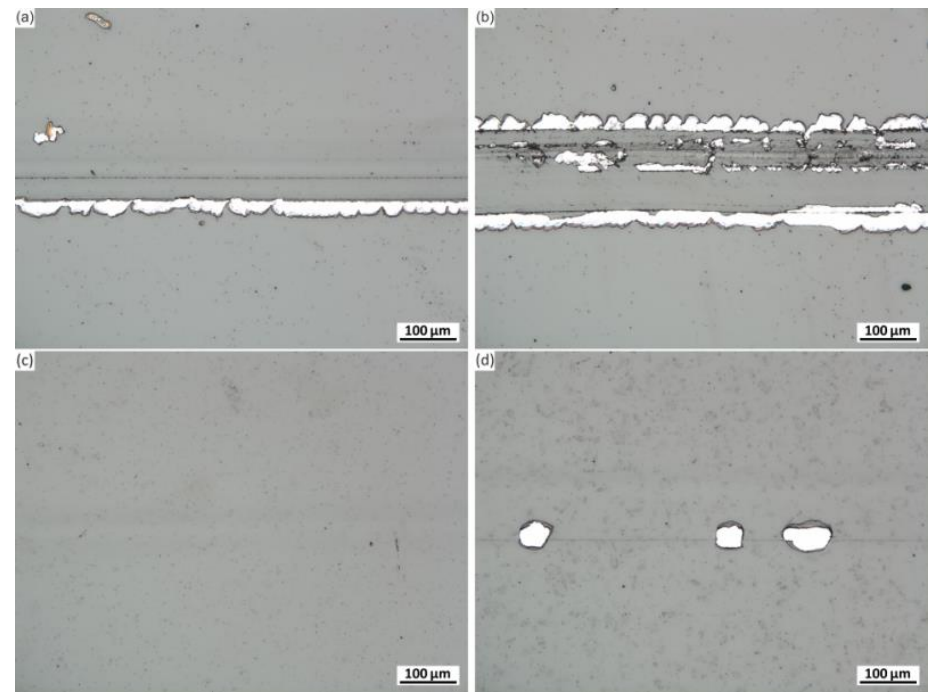

Figura 12: Micrografías ópticas de las huellas de Scratch Test: Muestra recubierta con $20 \mathrm{~N}$ (a) y $35 \mathrm{~N}$ (b). Las figuras c y d corresponden a la muestra dúplex con $20 \mathrm{~N}$ y $35 \mathrm{~N}$ respectivamente. 
También se realizaron ensayos de adhesión con indentación Rockwell C con una carga de $300 \mathrm{~N}$ en la muestra dúplex y recubierta. Se aprecia en la Figura 13, que la muestra recubierta presenta una región donde el recubrimiento se desprendió alrededor de la indentación. En la muestra dúplex, se observaron fisuras alrededor de la indentación y una pequeña región de desprendimiento.
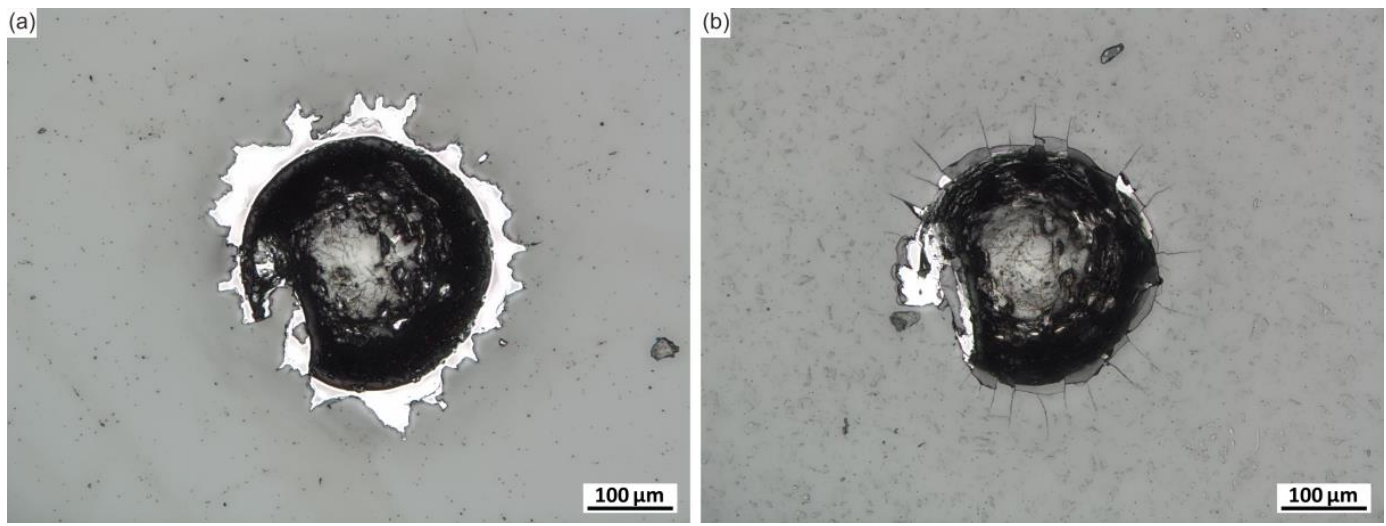

Figura 13: Micrografia ópticas de las huellas de indentaciones Rockwell C con $30 \mathrm{~kg}$ de carga. a) muestra recubierta, b) muestra dúplex.

En esta prueba, el indentador de diamante penetra en el recubrimiento induciendo una deformación plástica masiva del sustrato y la subsecuente fractura del recubrimiento. Como la capa nitrurada mejora la capacidad de carga de la zona superficial, puede prevenir la deformación plástica y fractura, resultando en una mejor adhesión [21].

En ambos ensayos, la muestra dúplex presentó mejor adhesión. Esto podría deberse a que la capa nitrurada, proporciona una interfase de transición gradual entre el recubrimiento DLC y el sustrato, causando una reducción de tensiones y así mejorando la adhesión, como fue reportado por otros autores [23,24].

No obstante, es posible que haya cierta afinidad química entre el silicio de la intercapa y el nitrógeno de la capa nitrurada, produciendo una unión química entre ambos elementos y mejorando la adhesión entre el recubrimiento y el sustrato[22].

\section{CONCLUSIONES}

Los recubrimientos DLC "hard" tuvieron una dureza de aproximadamente 25 GPa y un espesor de 1,4 $\mu \mathrm{m}$. El recubrimiento redujo el coeficiente de fricción respecto de la muestra nitrurada y sin tratar y mejoró la resistencia al desgaste.

La capa nitrurada previa a la deposición del recubrimiento mejoró la adhesión, verificado en los ensayos de Scratch Test e indentación.

Los recubrimientos presentaron buen comportamiento a la corrosión en el ensayo de niebla salina, en los ensayos propuestos en la Norma F 1089-02 y en las pruebas potenciostáticas. Estos recubrimientos podrían constituir una buena opción para proteger aceros para instrumentos quirúrgicos.

\section{AGRADECIMIENTOS}

Ala Ing. Catherine Aristizábal por el uso del SEM-FIB de la Universidad del Saarland, Saarbrücken, Alemania. A Vanesa Fuchs de la Universidad Nacional de Mar del Plata por la realización de los experimentos de difracción de rayos X. A la Dra. Adriana Márquez (INFIP-UBA) por el uso del Scratch Test. Al Ing. Mauro Moscatelli por los ensayos de corrosión y a todos los becarios alumnos del Grupo GIS (UTN) por su colaboración en preparación de muestras y realización de ensayos.

\section{BIBLIOGRAFÍA}

[1] SANCHEZ-SARRÍA, O., GONZALEZ-DIEZ, Y., HERNÁNDEZ DÁVILA, C., et al., "Manual de instrumental quirúrgico",Medisur, v. 12, n. 5, pp. 781- 818, Oct. 2014.

[2] MARCINIAK, J., PASZENDA, Z., WALKE, W., et al., "DLC coatings on martensitic steel used for surgical instruments”,Archives of Materials Science and Engineering, v.28, n. 5, pp. 285-288, May. 2007. 
[3] NARAYAN, R., "Diamond-Based materials for biomedical applications", Woodhead Publishing Limited, March 2013.

[4] JONES, B.J., MAHENDRAN, A., ANSON, A.W., et al., "Diamond-like carbon coating of alternative metal alloys for medical and surgical applications", Diamond and Related Materials, v. 19, n. 7-9, pp. 685689, Feb. 2010.

[5] ALPHONSA, I., HAINANI, A., RAOLE, P.M., et al., "A study of martensitic stainless steel AISI 420 modified using plasma nitriding”, Surface and Coatings Technology, v. 150, n. 2-3, pp. 263-268, Feb. 2002.

[6] CORENGIA, P., YBARRA, G., MOINA, C., et al., "Microstructure and corrosion behaviour of DCpulsed plasma nitrided AISI 410 martensitic stainless steel", Surface and Coatings Technology, v.187, n. 1, pp. 63-69, Oct. 2004.

[7] LI, C.X., BELL, T., "Corrosion properties of plasma nitrided AISI 410 martensitic stainless steel in 3.5\% $\mathrm{NaCl}$ and 1\% $\mathrm{HCl}$ aqueous solutions”, Corrosion Science, v. 48, n. 8, pp. 2036-2049, Aug. 2006.

[8] ROBERTSON, J., "Diamond-like amorphous carbon”, Materials Science and Engineering: R: Reports, v 37, n. 4-6,pp. 129-281, May 2002.

[9] ERDEMIR, A., DONNET, C., "Tribology of diamond-like carbon films: recent progress and future prospects", Journal of Physics D: Applied Physics, v. 39, n. 18, pp. R311-R327, Sept. 2006.

[10] GRILL, A., "Diamond-like carbon: state of the art", Diamond and Related Materials, v. 8, n. 2-5, pp. 428-434,March. 1999.

[11] BELL, T., DONG, H., SUN, Y., "Realising the potential of duplex surface engineering", Tribology International, v. 131, n. 1-3, pp. 127-137, Jan. 1998.

[12] SNYDERS, R., BOUSSER, E., AMIREAULT, P., et al., "Tribo-Mechanical Properties of DLC Coatings Deposited on Nitrided Biomedical Stainless Steel”, Plasma Process. and Polymers, v. 4, S1, pp. S640 S646, April 2007.

[13] AZZI, M., PAQUETTE, M., SZPUNAR, J.A., et al., "Tribocorrosion behaviour of DLC-coated 316L stainless steel”, Wear, v. 267, n. 5-8, pp. 860-866, Jun. 2009.

[14] ASTM F1089-2 “Standard Test Method for Corrosion of Surgical Instruments”, 2002.

[15] CAPOTE, G., BONETTI, L.F., SANTOS, L.V., et al., “Adherent amorphous hydrogenated carbon films on metals deposited by plasma enhanced chemical vapor deposition", Thin Solid Films, v. 516, n. 12, pp. 4011-4017, April 2008.

[16] ASTM Standard B117 “Standard Practice for Operating Salt Spray (Fog) Apparatus”, 1997.

[17] FERRARI, A.C., ROBERTSON, J., "Interpretation of Raman spectra of disordered and amorphous carbon”, Physical Review B, v. 61, pp. 14095-14107, May 2000.

[18] CASIRAGHI, C., FERRARI, A.C., ROBERTSON, J., "Raman spectroscopy of hydrogenated amorphous carbons", Physical Review B., v. 72,pp. 085401-13, Aug. 2005.

[19] XI, Y., LIU, D., HAN, D., "Improvement of corrosion and wear resistances of AISI 420 martensitic stainless steel using plasma nitriding at low temperature", Surface and Coatings Technology, v. 202, n. 12, pp. 2577-2583, March 2008.

[20] JEDRZEJOWSKI, P., KLEMBERG-SAPIEHA, J.E., MARTINU, L., "Relationship between the mechanical properties and the microstructure of nanocomposite TiN/SiN1.3 coatings prepared by low temperature plasma enhanced chemical vapor deposition", Thin Solid Films, v. 426, n. 1-2 pp.150-159, Feb. 2003.

[21] GRILL, A., “Tribology of diamondlike carbon and related materials: an updated review", Surface and Coatings Technology, v. 94-95, pp. 507-513, Oct. 1997.

[22] KWIETNIEWSKI, T.H.C., FONTANA, W., MORAES, C., et al., "Nitrided layer embrittlement due to edge effect on duplex treated AISI M2 high-speed steel”, Surface and Coatings Technology, v. 179, n. 1, pp. 27-32, Feb. 2004.

[23] GIL, L., BRÜHL, S., JIMÉNEZ, L., et al., "Corrosion performance of the plasma nitrided 316L stainless steel”, Surface and Coatings Technology, v. 201, n. 7, pp. 4424-4429, Dec. 2006.

[24] DALIBON, E.L., BRÜHL, S.P., TRAVA-AIROLDI, V.J., et al., "Hard DLC coating deposited over nitrided martensitic stainless steel: analysis of adhesion and corrosion resistance", Journal of Materials Research, v. 31, n. 22, pp. 3549-3556, Nov. 2016. 